\title{
Puerperal complications following elective cesarean sections for twin pregnancies
}

\author{
Teresinha Simões', Leonor Aboim¹, Ana Costa1, \\ Alexandre Ambrosio', Sandra Alves ${ }^{1}$ and Isaac \\ Blickstein $^{2}$ \\ ${ }^{1}$ Department of Maternal-Fetal Medicine, Maternity \\ Dr. Alfredo da Costa, Lisbon, Portugal \\ 2 Obstetrics and Gynecology, Kaplan Medical Center, \\ Rehovot and the Hadassah-Hebrew University School \\ of Medicine, Jerusalem, Israel
}

\begin{abstract}
Objective: To estimate the maternal puerperal morbidity in elective and emergent cesareans in twins.

Study design: We evaluated postpartum complications among patients who underwent elective cesarean birth for twin pregnancy. This group was compared to matched singletons and to emergent cesareans in twins. Results: During the period September 1994-March 2006 there were 299 (47.4\%) elective and 80 (12.7\%) emergent cesarean sections in twin pregnancies, for a total of 379 $(60.1 \%)$ cesarean births for both twins. Controls included 299 cases of elective cesareans in singletons. The comparison between elective and emergent cesareans and between elective cesareans in twins and in singletons found no significant differences in postpartum fever, scar infection, and postpartum hemorrhage. Venous thromboembolism occurred in two twin pregnancies, one in the elective and one in the emergent cesarean group. Postpartum hysterectomy was required in a singleton pregnancy following an elective cesarean birth.

Conclusion: At present, no data exist to show a disadvantage for a planned cesarean birth for twins.
\end{abstract}

Keywords: Cesarean; postpartum; puerperal morbidity; singletons; twins.

\section{Introduction}

Current efforts to diminish the escalating numbers of multiple pregnancies effectively reduced the incidence of higher-order multiples [4, 9]. At the same time, however, the number of twins is still increasing. The most recent

\footnotetext{
${ }^{\star}$ Corresponding author:

Isaac Blickstein, MD

Department of Obstetrics and Gynecology

Kaplan Medical Center, 76100 Rehovot, Israel

Phone: +972-8-9441930

Fax: +972-8-9441137

E-mail: blick@netvision.net.il
}

USA data indicate that twin birth rate increased $2 \%$ in 2004, to 32.3 twins per 1000 births, another all-time record high. The twinning rate has increased $42 \%$ since 1990 and $70 \%$ since 1980 . The most recent available national data from the USA indicate that as many as 132,219 twin births occurred in 2004 [6]. A similar trend has been observed in the United Kingdom as well as in other developed countries [4, 9]

As the numbers of twins increase, the mode of delivery becomes more pertinent. The United States cesarean birth rates increased 13\% (from 51.9 to $55.0 \%, 95 \% \mathrm{Cl}$ 12-14) between 1989-1991 and 1997-1999 among twins delivered at $\geq 22$ weeks and weighing $\geq 500 \mathrm{~g}$ [2]. Although this rate does not include the period after the publication of the Term Breech Trial [5], it represents an average increase of 52, 28 and $9 \%$ among twin pregnancies delivered at 22-27 weeks', 28-33 weeks' and at $\geq 34$ weeks' gestation, respectively. It was rightfully noted that the rates increased to a greater extent at earlier rather than at later gestational ages, but the absolute number of cesareans was much higher at later gestational ages [2]. These figures are quite similar to the commonly cited rates of $50-60 \%$ abdominal births among twins and nearly $100 \%$ among triplets [3]. In the UK, the 2001 cesarean rate for twin deliveries was 59\% [8].

At present, many of the circumstances that may have led to a twin pregnancy are commonly used as an indication for an elective cesarean delivery of twins. It appears that patients, as well as their attending clinicians, may base their decision for a cesarean in such "premium" pregnancies, intentionally or not, on quantitative arguments that are difficult to interpret and on qualitative variables that are impossible to quantify [3].

These considerations are contrasted with surprisingly scant information about puerperal morbidity following a planned compared to an emergent cesarean birth for twins, and compared with cesareans in singletons [1, 10]. Such information might be an important argument in the continuing discussion about the preferred mode of delivery of twins. The purpose of this paper was to estimate the maternal puerperal morbidity in elective and emergent cesareans in twins.

\section{Materials and methods}

During the period September 1994-March 2006, there were 946 twin pregnancies followed and delivered at the Maternity Dr. Alfredo da Costa, Lisbon, Portugal. This figure represents 
nearly $1 \%$ of all deliveries. During this period, information about the pregnancy and delivery was prospectively registered on a preset form and subsequently entered into a computerized system. This study focused on patients delivered by elective cesarean section as compared with emergent cesareans (defined as a decision taken during trial of labor for both twins). Thus, cases of cesareans for the second twin only-the so-called "combined twin delivery"-were excluded from the analysis. In addition, we focused on deliveries at $\geq 32$ weeks, to avoid the confounding effects of indications for very preterm cesareans. Finally, all cases with premature rupture of membranes, irrespective of the a priori planned mode of delivery, were counted as emergency cesareans.

For a secondary comparison, we created a new dataset of a matched cohort of singletons, comprising the successive singleton pregnancy that had a planned, elective, cesarean birth performed at $\geq 32$ weeks. Obviously, the indication for elective cesarean deliveries in twins and singleton differed. However, these indications are not associated with increased risk of puerperal morbidity that comprises the study variables listed below. The matching process assumes that the consecutive planned cesarean delivery in singletons within the same gestational age limits is the best randomly selected matched control for every case of elective cesarean in twins.

The following variables were compared: maternal age, parity, mode of conception, gestational age at birth, and birth weight. The study variables of interest were postpartum fever (defined as $>38^{\circ} \mathrm{C}$, measured twice, at $24 \mathrm{~h}$ postpartum or later), scar infection (defined as evident infection occurring during hospitalization, requiring either of the following measures: drainage, exploration of the scar, and antibiotic therapy), postpartum hemorrhage (defined as the need for blood transfusion or for active intervention to stop bleeding), and venous thromboembolism.

Our intraoperative protocol of antibiotic therapy ( $2 \mathrm{~g}$ of cephazoline, or an equivalent for allergic patients) was given following clamping of the singleton and the second twin's umbilical cord. Similarly, and irrespective of plurality, all cases received the same protocol of dilute oxytocin infusion. All cesareans were performed by senior staff. As noted above, in our service, rupture of membranes occurring in a patient scheduled for an elective cesarean changes her status to an emergent case.
The data were evaluated using the Microsoft Excel ${ }^{\circledR}$ program (Microsoft Corporation, Redmond, Washington). Comparisons were made between maternal variables of the three groups, but gestational age and birth weight variables were done between the twin groups only. We used the True epistat Software (Math Archives, Round Rock, TX) to perform Student's $t$ and Fisher's exact tests to compare continuous and categorical variables, respectively. We derived $\mathrm{P}$-values (considered significant if $<0.05$ ) and odds ratios (OR) and 95\% confidence interval $(\mathrm{Cl})$ for these comparisons. The study was approved by the local Ethical Committee.

\section{Results}

During the study period, there were 631 women delivered at $\geq 32$ weeks, including $299(47.4 \%)$ elective and 80 $(12.7 \%)$ emergent cesarean sections in twin pregnancies, for a total of $379(60.1 \%)$ cesarean births for both twins. We excluded $15(2.4 \%)$ cases of combined twin delivery for a total of $237(37.5 \%)$ vaginal births for both twins. Controls included 299 cases of elective cesareans in singletons.

Table 1 shows the comparison between the study and control groups. The mean maternal age in the three groups was similar; however, mothers who had an elective cesarean in singletons were more frequently over 35 years. Nulliparas were more frequent in twins compared to singletons and, as expected, there was a significantly lower frequency of spontaneous conceptions among twins compared to singletons. No difference was found in the comparison of the neonatal characteristics within the twin groups.

The comparison of the postpartum complications (Table 2) showed no significant difference between elective and emergent cesareans in twins and between elective cesareans in twins and singletons. Despite the insignificant differences it seems that elective cesareans

Table 1 Maternal and neonatal outcomes. Data presented as mean \pm SD or as $\mathrm{n}(\%)$.

\begin{tabular}{|c|c|c|c|}
\hline & \multicolumn{2}{|l|}{ Twins } & \multirow{2}{*}{$\begin{array}{l}\text { Singletons } \\
\text { Elective cesareans }\end{array}$} \\
\hline & Elective cesareans & Emergent cesareans & \\
\hline$n$ & 299 & 80 & 299 \\
\hline Maternal age (years) & $30.9 \pm 5.1$ & $29.8 \pm 5.3$ & $31.3 \pm 6.3$ \\
\hline Age $>$ 35 & $51(17.0)$ & $10(12.5)$ & 95 (31.8) \\
\hline Nulliparas $§$ & $158(52.8)$ & $47(58.7)$ & $82(27.4)$ \\
\hline Spontaneous pregnancies ${ }^{\star}$ & 231 (77.2) & $68(85.0)$ & $289(96.6)$ \\
\hline Gestational age (weeks) & $35.9 \pm 1.4$ & $35.8 \pm 1.6$ & $38.6 \pm 2.4$ \\
\hline$<35$ weeks & $52(17.3)$ & $18(22.5)$ & $18(6.0)$ \\
\hline Total birth weight (g) & $4799 \pm 726$ & $4807 \pm 751$ & $3239 \pm 750$ \\
\hline$<1500 \mathrm{~g}$ & $14 / 598(2.3)$ & 3/160 (1.9) & 10/299 (3.3) \\
\hline$<2500 \mathrm{~g}$ & $346 / 598$ (57.8) & $91(56.9)$ & $35 / 299$ (11.7) \\
\hline
\end{tabular}

"Twins vs. singletons OR $0.4(95 \% \mathrm{Cl} 0.3,0.7)$.

§Twins vs. singletons OR $3.0(95 \% \mathrm{Cl} 2.1,4.2)$.

${ }^{*}$ Twins vs. singletons OR $0.1(95 \% \mathrm{Cl} 0.05,0.2)$. 
Table 2 Puerperal complications. Data are shown as $n(\%)$, statistics are shown as OR (95\% Cl).

\begin{tabular}{llll}
\hline & Twins & Singletons & Statistics \\
\hline Postpartum fever & $7(2.3)$ & $6(2.0)$ & $1.2(0.3,4.0)$ \\
$\quad$ Elective & $5(6.3)$ & & \\
Emergent & $0.3(0.1,1.3)$ & & \\
Statistics & & $2(0.7)$ & $3.0(0.5,14.1)$ \\
Scar infection & $6(2.0)$ & & \\
Elective & $4(5.0)$ & $3(1.0)$ & $3.8(0.9,12.5)$ \\
Emergent & $0.4(0.1,1.7)$ & & \\
Statistics & & & \\
Postpartum hemorrhage & $11(3.7)$ & & \\
Elective & $3(3.8)$ & & \\
Emergent & $1.0(0.2,3.6)$ & & \\
Statistics & & & \\
\hline
\end{tabular}

in twins have a lower incidence of postpartum fever and scar infection compared to emergency cesareans in twins or elective cesareans in singletons. Postpartum venous thromboembolism occurred in two twin pregnancies, one in the elective and one in the emergent cesarean group. Of note is that postpartum hysterectomy was required in one case of hemorrhage in a singleton pregnancy following an elective cesarean birth.

\section{Discussion}

A recent analysis of epidemiological data by Meyer [7] found that 97 cesarean sections would be required to prevent a serious morbidity or mortality in a second twin. This number was within the range needed to prevent uterine rupture during a trial of labor following a cesarean $(1: 556)$ or morbidity related to vaginal breech delivery (1:167). Meyer rightfully pointed out that the current balance of risks related to cesarean birth in twins is incomplete because the potential risk of cesarean birth is practically unknown. Indeed, post-cesarean maternal complications are relatively rare and potentially underreported in epidemiological datasets.

Bearing in mind the potential type II (beta) error in the statistical analysis of data from a single center and from a relatively short period of observation, we used a carefully selected matched cohort that found that the complication rates among elective cesareans in twins were similar to those in emergent cesareans in twins and in elective cesareans in singletons. At the same time, however, a trend could be seen, whereby the frequencies of complications were 2-4 times higher in twins than in singletons (Table 2).

Our data are unable to confirm the origin of postpartum fever reported by Suonio and Huttunen [10] who evaluated the infectious complications of 122 consecutive cesarean twin births in Finland. These authors found that the incidence of endometritis and wound infection were nearly thrice and twice higher in twins compared with singleton cesarean deliveries, respectively. The authors identified young maternal age ( $<25$ years) and a prolonged interval between PROM and delivery ( $>6 \mathrm{~h}$ ) as risk factors for puerperal endometritis among twins, but a distinction between elective and emergent cesareans was not clearly defined. The increased puerperal infectious morbidity shown by this Finnish group was supported by Alexander et al. [1] who found a relatively high rate of metritis (18\%) among their cesarean sections performed in twins. Importantly, the hypothesis proposed by Suonio and Huttunen [10], suggesting that the larger placental bed in twins might be more susceptible to endometritis and thus leading to puerperal infectious morbidity could not be confirmed by our much larger data set.

We conclude that, at present, no solid data exist to show a disadvantage of a planned cesarean birth for twins. Having said this may not suggest that all twins should be delivered by cesarean section, but just to question the concerns that were raised regarding elective cesareans in twins. However, the trend of increased febrile puerperal morbidity following cesarean birth in twins requires further confirmation.

\section{References}

[1] Alexander JM, LC Gilstrap 3 $3^{\text {rd }}$, SM Cox, SM Ramin: The relationship of infection to method of delivery in twin pregnancy. Am J Obstet Gynecol 177 (1997) 1063

[2] Ananth CV, KS Joseph: Impact of obstetric intervention on trends in perinatal mortality. In Blickstein I, Keith LG, eds. Multiple Pregnancy: Epidemiology, Gestation, and Perinatal Outcome, 2nd edn. London: Taylor and Francis, (2005) 651

[3] Blickstein I: Cesarean section for all twins? J Perinat Med 28 (2000) 169

[4] Blickstein I, LG Keith: The decreased rates of triplet births: temporal trends and biologic speculations. Am J Obstet Gynecol 193 (2005) 327 
[5] Hannah ME, WJ Hannah, SA Hewson, ED Hodnett, S Saigal, AR Willan: Planned caesarean section versus planned vaginal birth for breech presentation at term: a randomised multicentre trial. Term Breech Trial Collaborative Group. Lancet 356 (2000) 1375

[6] Martin JA, BE Hamilton, PD Sutton, SJ Ventura, F Menacker, S Kirmeyer: Births: final data for 2004. Natl Vital Stat Rep 55 (2006) 1

[7] Meyer MC: Translating data to dialogue: How to discuss mode of delivery with your patient with twins. Am J Obstet Gynecol 195 (2006) 899

[8] Royal College of Obstetricians and Gynecologists. The National Sentinel Caesarean Section Audit Report, Octo- ber 2001, RCOG Clinical Effectiveness Support Unit, London

[9] Simmons R, P Doyle, N Maconochie: Dramatic reduction in triplet and higher order births in England and Wales. $\mathrm{Br}$ J Obstet Gynaecol 111 (2004) 856

[10] Suonio S, M Huttunen: Puerperal endometritis after abdominal twin delivery. Acta Obstet Gynecol Scand 73 (1994) 313

Received November 18, 2006. Revised January 5, 2007. Accepted January 8, 2007. 
Copyright of Journal of Perinatal Medicine is the property of Walter de Gruyter GmbH \& Co. KG. and its content may not be copied or emailed to multiple sites or posted to a listserv without the copyright holder's express written permission. However, users may print, download, or email articles for individual use. 
Copyright of Journal of Perinatal Medicine is the property of Walter de Gruyter GmbH \& Co. KG. and its content may not be copied or emailed to multiple sites or posted to a listserv without the copyright holder's express written permission. However, users may print, download, or email articles for individual use. 